\title{
Molecular Genetic Characterization of Acute Myeloid Leukemia With Trisomy 4 as the Sole Chromosome Abnormality
}

\author{
SYNNE TORKILDSEN ${ }^{1,2}$, LUDMILA GORUNOVA $^{1}$, SVERRE HEIM $^{1,3}$, GEIR E. TJØNNFJORD ${ }^{2,3}$, \\ SIGNE SPETALEN $^{4}$, BENTE RISBERG ${ }^{4}$, HOA THI TUYET TRAN ${ }^{5}$ and IOANNIS PANAGOPOULOS ${ }^{1}$ \\ ${ }^{1}$ Section for Cancer Cytogenetics, Institute for Cancer Genetics and Informatics, \\ Oslo University Hospital, Oslo, Norway; \\ ${ }^{2}$ Department of Haematology, Oslo University Hospital, Oslo, Norway; \\ ${ }^{3}$ Institute of Clinical Medicine, University of Oslo, Oslo, Norway; \\ ${ }^{4}$ Department of Pathology, University of Oslo, Oslo, Norway; \\ ${ }^{5}$ Department of Haematology, Akershus University Hospital, Lørenskog, Norway
}

\begin{abstract}
Background/Aim: The aim of the study was to determine the genetic and molecular consequences of trisomy 4, a recurrent but rare chromosomal abnormality in acute myeloid leukemia (AML). Materials and Methods: Interphase fluorescence in situ hybridization, reverse transcriptasequantitative polymerase chain reaction for 28 chromosomal gene translocations/fusion genes, and targeted sequencing analyses were performed on five AMLs with trisomy 4 as the sole chromosomal anomaly. Results: An NPM1 frameshift mutation was found in all leukemic bone marrows, DNMT3A, FLT3, and IDHI mutations were found in three, KIT and NRAS mutations in two, whereas IDH2 (RI40Q), RUNXI, and WTI mutations were found in only one patient each. The three patients with a DNMT3A (R882H) mutation have died. In contrast, the two patients whose leukemic cells were without this mutation, are alive 55 and 31 months after diagnosis, respectively. Conclusion: The results suggest a possible association between trisomy 4 and additional mutations that may influence prognosis.
\end{abstract}

Acute myeloid leukemia (AML) is a heterogenous group of malignancies with different clinical phenotypes and responses to therapy $(1,2)$. AMLs can be classified according to distinct cytogenetic and genetic abnormalities of leukemic cells at

This article is freely accessible online.

Correspondence to: Ioannis Panagopoulos, Section for Cancer Cytogenetics, Institute for Cancer Genetics and Informatics, The Norwegian Radium Hospital, Oslo University Hospital, Montebello, PO Box 4954 Nydalen, NO-0424 Oslo, Norway. Tel: +47 22782362, e-mail: ioannis.panagopoulos@rr-research.no

Key Words: Acute myeloid leukemia, trisomy 4, targeted sequencing, mutations, NPM1. diagnosis, but also by phenotypic and epigenetic differences that act as a guide to risk assessment and choice of treatment (1, 2). AML cases with a single trisomy constitute a heterogenous subgroup with regard to clinical, morphological, and immunophenotypic features, as well as the mutational profiles (3).

Trisomy 4 is a recurrent, but rare chromosomal abnormality in AML. It may be the sole aberration or one of several chromosomal changes (4). Trisomy 4 was first described by Mecucci et al. in 1986, and a year later by Sandberg et al. (5), as the sole chromosomal abnormality in AML (6). In the Mitelman Database of Chromosome Aberrations and Gene Fusions in Cancer, 83 (48 female and 35 male patients) out of 18029 AML-cases had trisomy 4 as the sole chromosomal abnormality (http://cgap.nci.nih.gov/Chromosomes/Mitelman, database last updated on February 20, 2017). Most cases are morphologically classified as M1, M2, or M4 using the FrenchAmerican-British (FAB) classification.

Because trisomy 4 is rare, few studies have addressed its clinical impact. As a consequence, the prognosis of AML with solitary trisomy 4 remains unclear $(7,8)$. However, a recent study based on 87 AML cases with trisomy 4 concluded that the prognostic significance of the aberration depended on the patient's age, and despite an initial good response to treatment, patients with trisomy 4 were prone to relapse (4).

Recently, we reported the genetic, molecular and clinical features of a pediatric AML case with trisomy 4 and an FLT3ITD mutation (9). Fluorescence in situ hybridization assay demonstrated that part of the RUNXI probe had moved to chromosome band $6 \mathrm{q} 25$ indicating a cryptic $\mathrm{t}(6 ; 21)(\mathrm{q} 25 ; \mathrm{q} 22)$ translocation. Molecular analysis of the translocation showed fusion of RUNX1 with an intergenic sequence from $6 \mathrm{q} 25$, resulting in a putative RUNX1 truncated protein that would contain the Runt homology domain responsible for both heterodimerization with CBFB and DNA binding (9). The findings prompted us to investigate additional cases of myeloid 
Table I. Clinicopathological and hematological findings of AML patients with trisomy 4 as the sole chromosome abnormality.

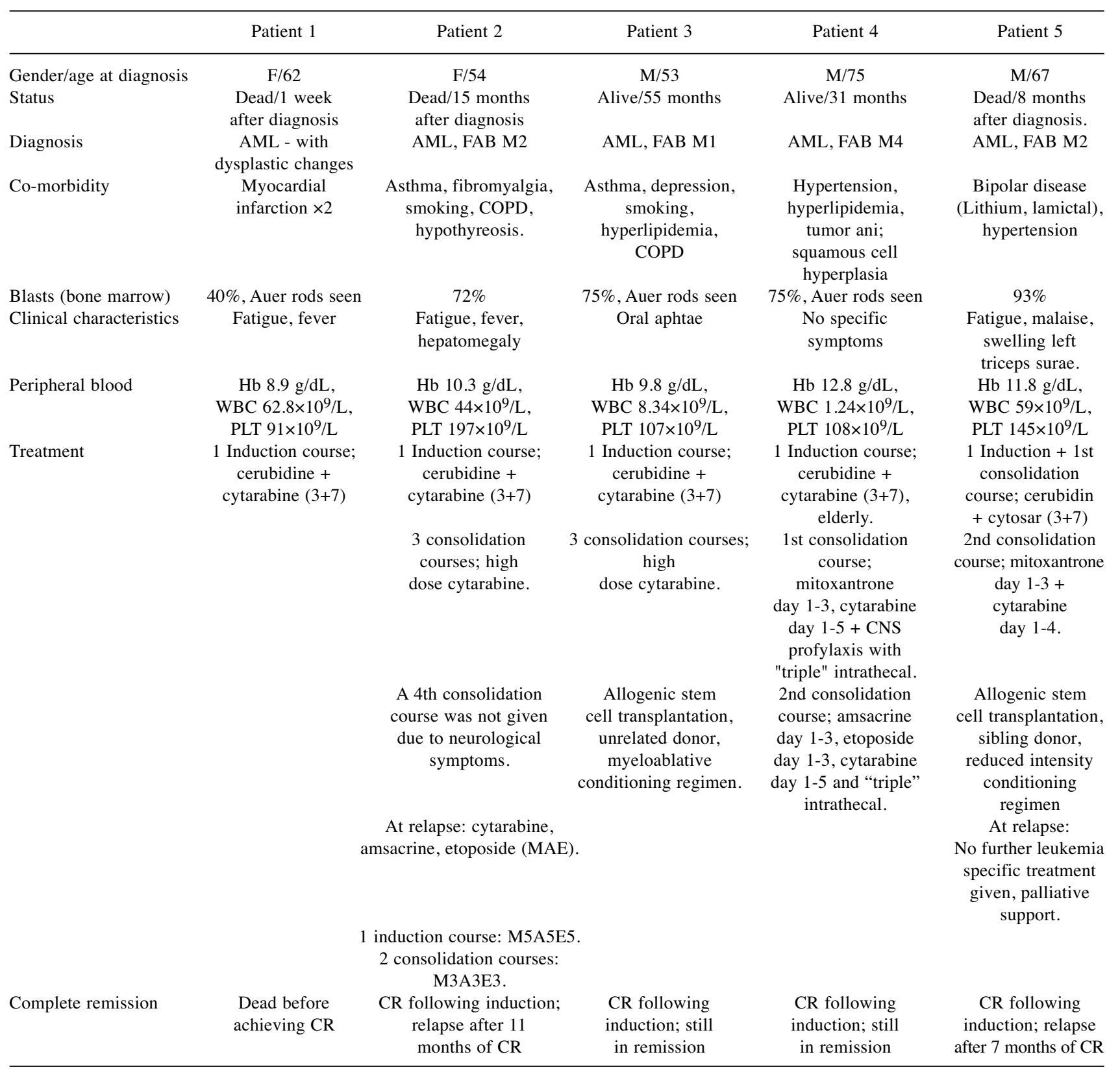

AML: Acute myeloid leukemia; COPD: chronic obstructive pulmonary disease; Hb: hemoglobin; PLT: platelet count; WBC: white blood cell count; CR: complete remission.

malignancies with trisomy 4 as the sole karyotypic anomaly, for submicroscopic or molecular aberrations.

\section{Materials and Methods}

Patients and samples. Permission to perform the study was granted by the Regional Committee for Medical and Health Research Ethics of South-East Norway, and written informed consent was obtained from the patients.
Searching our archive of 5700 cytogenetically analyzed leukemia samples (from 2009 until 2017) five additional cases of AML with trisomy 4 as the sole anomaly were found (Table I).

Methods. Interphase fluorescence in situ hybridization (FISH) was performed on these samples with the Cytocell multiprobe AML/MDS panel (Cytocell, http://www.cytocell.co.uk/) looking for $\operatorname{del}(5 \mathrm{q})$, PML/RARA, del(17p) (TP53), RUNX1/RUNX1T1, trisomy 8, KMT2A (MLL) splitting, -7/del(7q), CBFB/MYH11, and $\operatorname{del}(20 \mathrm{q})$. The HemaVision 28Q reverse transcription-quantitative polymerase chain 
Torkildsen et al: Genetic Characterization of Acute Myeloid Leukemia With Trisomy 4

Table II. Mutational profile after exome sequencing of AML patients with trisomy 4 as the sole chromosome abnormality.

\begin{tabular}{lccccc}
\hline Genes & Patient 1 & Patient 2 & Patient 3 & Patient 4 & Patient 5 \\
\hline NPM1 & W288fs*12 & W288fs*12 & W288fs*12 & W288fs*12 & W288fs*12 \\
DNMT3A & R882H & R882H & G12S & & R882H \\
NRAS & G12D & & D835Y & & E598DYVDFREYE \\
FLT3 & D835Y & D816V & & R132H & D816V \\
KIT & & R132H & R140Q & & R132L \\
$I D H 1$ & & E223G & D464N & S480G \\
IDH2 & & & & \\
RUNX1 & & T58I & & \\
CUX1 & & & &
\end{tabular}

reaction (RT-qPCR-KIT) for 28 chromosome translocations/ fusion genes (DNA Diagnostics, Risskov, Denmark) was used to detect the most common AML-specific fusion transcripts.

The Illumina's TruSight Myeloid Sequencing Panel (Illumina, San Diego, CA, USA) was used to identify somatic mutations in the samples. According to the company's information, the TruSight Myeloid Sequencing Panel covers 15 full genes (exons only) and 39 additional genes that are oncogenic hotspots. Alignment and variant calling were performed against GRCh37/hg19 with MiSeq Reporter Software v.2.6.3.2 (Illumina). SNV's and indels $<60$ bp were annotated by VariantStudio v.2.2.

\section{Results}

Neither interphase FISH analyses found any aberrations nor HemaVision 28Q RT-qPCR-KIT analysis detected any of the most common AML-specific fusion transcripts.

Targeted sequencing showed that all five examined AML samples carried mutations in addition to trisomy 4 (Table II). Samples from patients 1, 2, 4, and 5 harbored four mutations whereas patient 3 had seven mutations. NPM1 frameshift mutation was found in all patients, whereas KIT and NRAS mutations were found in two patients. DNMT3A, FLT3, and $I D H 1$ mutations were found in three patients, whereas $I D H 2$ (R140Q), RUNX1, CUX1, KRAS, and WT1 mutations were found in one patient each. Patients 1,2, and 5, whose AMLs showed a DNMT3A (R882H) mutation, have died. In contrast, patients 3 and 4, whose leukemic cells did not carry this mutation, are alive 55 and 31 months after diagnosis, respectively. The leukemic cells from the deceased patients 2 and 5 also harbored the KIT D816V mutation, whereas patients 3 and 4 without this mutation are alive 55 and 31 months after diagnosis. Patient 1, also deceased, did not have the acquired KIT D816V mutation. This patient succumbed suddenly probably due to a cardiac event rather than AML.

\section{Discussion}

The genetic and molecular consequences of trisomy 4 are, in general, unknown $(3,4,7,8)$. Possible mechanisms involve global gene expression alterations because of gene dosage effects, including duplication of rearranged or mutated genes on chromosome 4 . No studies have as yet specifically examined general expression patterns in AML with trisomy 4. Instead, analyses have focused on $K I T$ at $4 \mathrm{q} 12$, which encodes a receptor tyrosine kinase related to FLT3. Ferrari et al. (10) reported overexpression of KIT in one case with trisomy 4 as compared to AML without this abnormality, suggesting a possible pathogenetic role. Beghini et al. (11) showed that trisomy 4 resulted in duplication of a mutated KIT allele in two out of six AML cases with trisomy 4 as the sole change. Similarly, Schnittger et al. (12) reported KIT mutations in two cases with trisomy 4. On the other hand, Bains et al. (7) studied 13 AML cases with isolated trisomy 4 finding a KIT mutation in only one of them, and concluded that trisomy 4 was not generally associated with KIT mutations. Instead, FLT3 and NPM1 mutations were found in $50 \%$ and $40 \%$ of the cases, respectively.

In the present study, targeted sequencing showed concurrent multiple mutations in all patients (Table II). An NPM1 frameshift mutation was found in all leukemic bone marrows. NPMI mutations are common in AML and are found in $20-30 \%$ of the cases (13). In addition, mutations were found in genes which are involved in the regulation of DNA methylation (DNMT3A, IDH1 and IDH2) and genes involved in cell signaling (FLT3, NRAS and KIT). RUNX1, $C U X 1, K R A S$, and $W T 1$ mutations were found in one patient each.

The three patients with a DNMT3A (R882H) mutation have died. In contrast, the two patients whose leukemic cells did not carry a DNMT3A mutation, are alive 55 and 31 months after diagnosis, respectively.

DNMT3A mutations are the most frequent recurrent gene mutations in AML after NPM1 and FLT3 mutations (14). The prognostic significance of DNMT3A mutations is thought to be adverse. DNMT3A mutations arise early in AML evolution and may persist during remission $(1,14)$. There is, however, no clear information today as to whether 
any particular relationship exists between trisomy 4 and mutations of DNMT3A, let alone whether any such combination impacts prognosis.

It is unclear whether the poor outcome for patients 1,2 , and 5 was influenced by additional mutation(s) in DNMT3A, $K I T, I D H 1$ or a combination of these changes. The results, nevertheless, suggest a possible association between trisomy 4 and additional mutations that may affect prognosis. Novel techniques, such as targeted sequencing used in this study, may help identify the critical genes and understand their role in leukemogenesis and their influence on prognosis. Such investigations in large groups of patients are needed to collect quality data for this purpose.

\section{Conflicts of Interest}

The Authors declare that they have no potential conflicts of interest.

\section{Authors' Contributions}

ST made hematological evaluations, treated patients, designed the research, and wrote the manuscript. LG interpreted the cytogenetics and FISH data. SH interpreted the cytogenetics and FISH data and wrote the manuscript. GET made hematologic evaluations, treated patients and wrote the manuscript. SS made hematopathologic evaluations and interpreted the targeted sequencing data. BR performed targeted sequencing and interpreted the data. HTTT made hematologic evaluations and treated patients. IP designed and supervised the research, interpreted the data and wrote the manuscript.

\section{Acknowledgements}

This work was supported by grants from the Norwegian Radium Hospital Foundation and the Department of Haematology, Oslo University Hospital.

\section{References}

1 Saultz JN and Garzon R: Acute myeloid leukemia: A concise review. J Clin Med 5, 2016. PMID: 26959069. DOI: 10.3390/ jcm5030033

2 Dohner H, Estey E, Grimwade D, Amadori S, Appelbaum FR, Buchner T, Dombret H, Ebert BL, Fenaux P, Larson RA, Levine RL, Lo-Coco F, Naoe T, Niederwieser D, Ossenkoppele GJ, Sanz M, Sierra J, Tallman MS, Tien HF, Wei AH, Lowenberg B and Bloomfield CD: Diagnosis and management of AML in adults: 2017 ELN recommendations from an international expert panel. Blood 129: 424-447, 2017. PMID: 27895058. DOI: 10.1182/blood-2016-08-733196

3 Lazarevic VL, Rosso A, Juliusson G, Antunovic P, Derolf AR, Deneberg S, Mollgard L, Uggla B, Wennstrom L, Wahlin A, Hoglund $\mathrm{M}$, Lehmann $\mathrm{S}$ and Johansson B: Incidence and prognostic significance of isolated trisomies in adult acute myeloid leukemia: A population-based study from the Swedish AML registry. Eur J Haematol 98: 493-500, 2017. PMID: 28152233. DOI: $10.1111 /$ ejh.12861
4 Chilton L, Hills RK, Burnett AK and Harrison CJ: The prognostic significance of trisomy 4 in acute myeloid leukaemia is dependent on age and additional abnormalities. Leukemia 30: 2264-2267, 2016. PMID: 27451976. DOI: 10.1038/leu.2016.200

5 Sandberg AA, Morgan R, Sait SN, Berger R, Flandrin G, Schrier $\mathrm{S}$ and Hecht F: Trisomy 4: an entity within acute nonlymphocytic leukemia. Cancer Genet Cytogenet 26: 117-125, 1987. PMID: 3470127.

6 Mecucci C, Van Orshoven A, Tricot G, Michaux JL, Delannoy $\mathrm{A}$ and Van den Berghe $\mathrm{H}$ : Trisomy 4 identifies a subset of acute nonlymphocytic leukemias. Blood 67: 1328-1332, 1986. PMID: 3697508.

7 Bains A, Lu G, Yao H, Luthra R, Medeiros LJ and Sargent RL: Molecular and clinicopathologic characterization of AML with isolated trisomy 4. Am J Clin Pathol 137: 387-394, 2012. PMID: 22338050. DOI: 10.1309/AJCP7ZC9YQERSKGX

8 Gupta V, Minden MD, Yi QL, Brandwein $\mathrm{J}$ and Chun $\mathrm{K}$ : Prognostic significance of trisomy 4 as the sole cytogenetic abnormality in acute myeloid leukemia. Leuk Res 27: 983-991, 2003. PMID: 12859991.

9 Panagopoulos I, Torkildsen S, Gorunova L, Ulvmoen A, Tierens A, Zeller B and Heim S: RUNX1 truncation resulting from a cryptic and novel $\mathrm{t}(6 ; 21)(\mathrm{q} 25 ; \mathrm{q} 22)$ chromosome translocation in acute myeloid leukemia: A case report. Oncol Rep 36: 24812488, 2016. PMID: 27667292. DOI: 10.3892/or.2016.5119

10 Ferrari S, Grande A, Zucchini P, Manfredini R, Tagliafico E, Rossi E, Temperani P, Torelli G, Emilia G and Torelli U: Overexpression of c-kit in a leukemic cell population carrying a trisomy 4 and its relationship with the proliferative capacity. Leuk Lymphoma 9: 495501, 1993. PMID: 7687917. DOI: 10.3109/10428199309145756

11 Beghini A, Ripamonti CB, Cairoli R, Cazzaniga G, Colapietro P, Elice F, Nadali G, Grillo G, Haas OA, Biondi A, Morra E and Larizza L: KIT activating mutations: Incidence in adult and pediatric acute myeloid leukemia, and identification of an internal tandem duplication. Haematologica 89: 920-925, 2004. PMID: 15339674.

12 Schnittger S, Kohl TM, Haferlach T, Kern W, Hiddemann W, Spiekermann K and Schoch C: KIT-D816 mutations in AML1ETO-positive AML are associated with impaired event-free and overall survival. Blood 107: 1791-1799, 2006. PMID: 16254134. DOI: 10.1182/blood-2005-04-1466.

13 Heath EM, Chan SM, Minden MD, Murphy T, Shlush LI and Schimmer AD: Biological and clinical consequences of NPM1 mutations in AML. Leukemia 31: 798-807, 2017. PMID: 28111462. DOI: 10.1038/leu.2017.30

14 Medinger M, Lengerke C and Passweg J: Novel prognostic and therapeutic mutations in acute myeloid leukemia. Cancer Genomics Proteomics 13: 317-329, 2016. PMID: 27566651. 